PROCEEDINGS OF THE

AMERICAN MATHEMATICAL SOCIETY

Volume 133, Number 8, Pages 2401-2408

S 0002-9939(05)07790-7

Article electronically published on March 4, 2005

\title{
ALMOST AUTOMORPHIC SOLUTIONS OF SEMILINEAR EVOLUTION EQUATIONS
}

\author{
JEROME A. GOLDSTEIN AND GASTON M. N'GUÉRÉKATA
}

(Communicated by Carmen C. Chicone)

\begin{abstract}
We are concerned with the semilinear differential equation in a Banach space $\mathbb{X}$,

$$
x^{\prime}(t)=A x(t)+F(t, x(t)), \quad t \in \mathbb{R},
$$

where $A$ generates an exponentially stable $C_{0}$-semigroup and $F(t, x): \mathbb{R} \times$ $\mathbb{X} \rightarrow \mathbb{X}$ is a function of the form $F(t, x)=P(t) Q(x)$. Under appropriate conditions on $P$ and $Q$, and using the Schauder fixed point theorem, we prove the existence of an almost automorphic mild solution to the above equation.
\end{abstract}

\section{INTRODUCTION}

Consider in a Banach space $(\mathbb{X},\|\cdot\|)$ the semilinear differential equation

$$
x^{\prime}(t)=A x(t)+F(t, x(t)), \quad t \in \mathbb{R},
$$

where the linear operator $A: D(A) \subset \mathbb{X} \rightarrow \mathbb{X}$ generates an exponentially stable $C_{0}$-semigroup $\mathcal{T}=(T(t))_{t \geq 0}$; that is, $\mathcal{T}$ satisfies the estimate

$$
\|T(t)\| \leq M e^{-\epsilon t},
$$

for some constants $M>0, \epsilon>0$ and all $t \geq 0$. Let $F: \mathbb{R} \times \mathbb{X} \rightarrow \mathbb{X}$ be jointly continuous. A mild solution to (1.1) is a function $x \in C(\mathbb{R}, \mathbb{X})$ satisfying the integral equation

$$
x(t)=T(t-a) x(a)+\int_{a}^{t} T(t-s) F(s, x(s)) d s
$$

for every $a \in \mathbb{R}$ and every $t \geq a$.

A fundamental problem is the existence of almost automorphic mild solutions to (1.1). Recently, G. M. N'Guérékata [5] showed, using the Banach fixed point theorem, that if

i) $F$ is Lipschitzian in $x \in \mathbb{X}$, uniformly in $t \in \mathbb{R}$, that is,

$$
\|F(t, x)-F(t, y)\| \leq L\|x-y\|
$$

for all $x, y \in \mathbb{X}$, and $t \geq 0$, and $L$ is sufficiently small, namely $L<\frac{\epsilon}{M}$, where $\epsilon$ and $M$ are as in (1.2), and

Received by the editors February 11, 2004 and, in revised form, April 12, 2004.

2000 Mathematics Subject Classification. Primary 34A05, 34K05, 47D60, 34G20.

(C)2005 American Mathematical Society 
ii) $F(t, x)$ is almost automorphic in $t \in \mathbb{R}$ for each $x \in \mathbb{X}$,

then problem (1.1) has a unique almost automorphic mild solution.

In this paper, we are going to prove the existence of almost automorphic mild solutions to (1.1), $F$ being not necessarily Lipschitzian. But first, let us recall some definitions.

Definition 1.1. A continuous function $f: \mathbb{R} \rightarrow \mathbb{X}$ is said to be almost automorphic if for every sequence of real numbers $\left(s_{n}^{\prime}\right)$, there exists a subsequence $\left(s_{n}\right)$ such that

$$
g(t)=\lim _{n \rightarrow \infty} f\left(t+s_{n}\right)
$$

is well defined for each $t \in \mathbb{R}$, and

$$
\lim _{n \rightarrow \infty} g\left(t-s_{n}\right)=f(t)
$$

for each $t \in \mathbb{R}$.

It is well known that the range $\mathcal{R}_{f}=\{f(t) \mid t \in \mathbb{R}\}$ of an almost automorphic function $f$ is relatively compact in $\mathbb{X}$, thus bounded in norm (see [6], Theorem 2.13). The function $g$ in the definition is also bounded and strongly measurable. Also, the set $A A(\mathbb{X})$ of all almost automorphic functions $f: \mathbb{R} \rightarrow \mathbb{X}$ equipped with the sup-norm

$$
\|f\|_{\infty}=\sup _{t \in \mathbb{R}}\|f(t)\|
$$

is a Banach space (see [6], page 20).

Also, given two Banach spaces $\left(\mathbb{X}_{1},\|\cdot\|_{1}\right)$ and $\left(\mathbb{X}_{2},\|\cdot\|_{2}\right), B\left(\mathbb{X}_{1}, \mathbb{X}_{2}\right)$ will denote the Banach space of bounded linear operators $L: \mathbb{X}_{1} \rightarrow \mathbb{X}_{2}, B C\left(\mathbb{R}, \mathbb{X}_{1}\right)$ is the Banach space of all continuous and bounded functions $f: \mathbb{R} \rightarrow \mathbb{X}_{1}$, and $B U C\left(\mathbb{R}, \mathbb{X}_{1}\right)$ is the Banach space of all bounded and uniformly continuous functions $f: \mathbb{R} \rightarrow \mathbb{X}_{1}$.

\section{Preliminaries}

In this paper $(\mathbb{Y},|\cdot|)$ will denote a Banach space algebraically contained in $\mathbb{X}$ such that the canonical injection $\mathbb{Y} \rightarrow \mathbb{X}$ is compact. An example of such a space $\mathbb{Y}$ is an abstract Sobolev space that we construct as follows:

Let $A$ be as in (1.1), (1.2). By (1.2), $0 \in \rho(A)$, so that the fractional powers $(-A)^{\alpha}, 0<\alpha<1$, are well defined. Also, since $0 \in \rho(A)$, the norm

$$
|f|=\left\|(-A)^{\alpha} f\right\|
$$

is equivalent to the graph norm

$$
\|f\|_{\alpha}=\left\|(-A)^{\alpha} f\right\|+\|f\| .
$$

Now we take $\mathbb{X}=L^{p}(\Omega)$, where $1<p<\infty$ and $\Omega \subset \mathbb{R}^{n}$ is a smooth bounded domain in $\mathbb{R}^{n}$. Let $A$ be a linear uniformly elliptic operator (with suitable boundary conditions), of order $2 m$. Then let $\mathbb{Y}$ be the domain of $(-A)^{\alpha}$ with norm $(2.1)$; we have

$$
W_{0}^{2 m \alpha, p}(\Omega) \subset \mathbb{Y} \subset W^{2 m \alpha, p}(\Omega)
$$

and the norm $|\cdot|$ in $\mathbb{Y}$ is equivalent to the usual norm in $W^{2 m \alpha, p}(\Omega)$. Also, the injection $\mathbb{Y} \rightarrow \mathbb{X}$ is compact in this case, by Sobolev embedding. 


\section{MAin Results}

Now let $\mathbb{Y}=D\left((-A)^{\alpha}\right)$, the domain of $(-A)^{\alpha}$, with norm

$$
|y|=\left\|(-A)^{\alpha} y\right\|, \quad y \in D\left((-A)^{\alpha}\right),
$$

where $0<\alpha<1$ is fixed. We get

$$
|T(t) y|=\left\|T(t)(-A)^{\alpha} y\right\| \leq M e^{-\epsilon t}\left\|(-A)^{\alpha} y\right\|=M e^{-\epsilon t}|y|
$$

for each $y \in \mathbb{Y}$ and every $t \geq 0$, by (1.2).

We also make the following assumptions:

$$
F(t, x)=P(t) Q(x), \quad \text { for all } t \in \mathbb{R}, x \in \mathbb{X},
$$

where $P(t) \in A A(\mathbb{Z})$ for each $t \in \mathbb{R}$ with $\mathbb{Z}=B(\mathbb{X}, \mathbb{Y}) ; P$ is continuous from $\mathbb{R}$ to $A A(\mathbb{Z})$, and $Q: B C(\mathbb{R}, \mathbb{X}) \rightarrow B C(\mathbb{R}, \mathbb{X})$ is continuous and satisfies the estimate

$$
\|Q \varphi\|_{\infty} \leq \mathcal{M}\left(\|\varphi\|_{\infty}\right)
$$

where $\|f\|_{\infty}:=\sup _{t \in \mathbb{R}}\|f(t)\|$ and $\mathcal{M} \in C\left(\mathbb{R}^{+}, \mathbb{R}^{+}\right)$satisfies

$$
\lim _{r \rightarrow \infty} \frac{\mathcal{M}(r)}{r}=0 \text {. }
$$

Note that $\mathcal{M}$ can be unbounded but must grow slower than a linear function. Let

$$
[P]:=\sup _{t \in \mathbb{R}}\|P(t)\|_{\mathbb{Z}}<\infty .
$$

Define $G: B C(\mathbb{R}, \mathbb{X}) \rightarrow B C(\mathbb{R}, \mathbb{Y})$ by

$$
(G \varphi)(t)=\int_{-\infty}^{t} T(t-s) F(s, \varphi(s)) d s .
$$

For $\varphi \in B C(\mathbb{R}, \mathbb{X})$, this integral exists. Indeed, we have

$$
\begin{aligned}
|(G \varphi)(t)| & \leq \int_{-\infty}^{t}|T(t-s) \| P(t) Q(\varphi(s))| d s \\
& \leq \int_{-\infty}^{t} M e^{-\epsilon(t-s)}[P] \mathcal{M}\left(\|\varphi\|_{\infty}\right) d s
\end{aligned}
$$

using (3.1), (3.3) and (3.5). Consequently

$$
\begin{aligned}
& |G \varphi|_{\infty}=\sup _{t \in \mathbb{R}}|(G \varphi)(t)| \\
& \leq M \epsilon^{-1}[P] \mathcal{M}\left(\|\varphi\|_{\infty}\right) .
\end{aligned}
$$

Continuity of $G$ is straightforward by virtue of continuity of both $P$ and $Q$. Thus we have

$$
G(B C(\mathbb{R}, \mathbb{X})) \subset B C(\mathbb{R}, \mathbb{Y}) .
$$

Finally, for $0<\delta \leq 1$, let

$$
B C^{\delta}(\mathbb{R}, \mathbb{Y}) \equiv\left\{f \in B C(\mathbb{R}, \mathbb{Y}):|f|_{\delta, \mathbb{Y}}<\infty\right\},
$$

where

$$
|f|_{\delta, \mathbb{Y}} \equiv \sup _{t \in \mathbb{R}}|f(t)|+\delta \sup _{t, s \in \mathbb{R}, t \neq s} \frac{|f(t)-f(s)|}{|t-s|^{\delta}} .
$$

With the norm $|\cdot|_{\delta, \mathbb{Y}}, B C^{\delta}(\mathbb{R}, \mathbb{Y})$ turns out to be a Banach space of all bounded Hölder continuous $\mathbb{Y}$-valued functions on $\mathbb{R}$ of Hölder exponent $\delta$. 
Proposition 3.1. The function $G$ defined above maps bounded sets of $B C(\mathbb{R}, \mathbb{X})$ into bounded sets of $B C^{\delta}(\mathbb{R}, \mathbb{Y})$ for any $\delta>0$ satisfying $\delta<\alpha$, where $0<\alpha<1$ is the exponent defining $\mathbb{Y}=D(-A)^{-\alpha}$.

Proof. The proof is basically a modification of the above remarks. Let $0<\beta<\alpha$. Then

$$
\begin{aligned}
|(G \varphi)(t)| & =\left|\int_{-\infty}^{t} T(t-s)(-A)^{\beta}(-A)^{-\beta} F(s, \varphi(s)) d s\right| \\
& \leq \int_{-\infty}^{t}\left|T(t-s)(-A)^{\beta}\right|\left|(-A)^{-\beta} P(s)\right| \mid Q(\varphi(s) \mid d s .
\end{aligned}
$$

Now, by semigroup theory (see for instance [4]), there exists a constant $M_{1}$ such that

$$
\left\|T(r)(-A)^{\beta}\right\| \leq \frac{M_{1} e^{-\epsilon r}}{r^{\beta}}
$$

for all $r>0$. Thus we obtain, as previously,

$$
\left|T(r)(-A)^{\beta}\right| \leq M_{1} e^{-\epsilon r} r^{-\beta}, \quad r>0 .
$$

Next, we observe that the function $s \mapsto(-A)^{-\beta} P(s)$ is a uniformly bounded function $\mathbb{R} \rightarrow B\left(\mathbb{X}, D\left((-A)^{\alpha-\beta}\right)\right.$. Indeed, it is the composition of $P(\cdot): \mathbb{R} \rightarrow$ $B\left(\mathbb{X}, D\left((-A)^{\alpha}\right)\right)$, which is bounded by $[P]$, with $(-A)^{-\beta}$, an isometry from $D\left((-A)^{\alpha}\right)$ onto $D\left((-A)^{\alpha-\beta}\right)$. Thus

$$
\sup _{t \in \mathbb{R}}\|P(t)\|_{B\left(\mathbb{X}, D\left((-A)^{\alpha-\beta}\right)\right)} \leq[P] .
$$

Now combining the estimates in (3.8) and (3.9), we deduce

$$
|(G \varphi)(t)| \leq \int_{-\infty}^{t} M_{1} e^{-\epsilon(t-s)}(t-s)^{-\beta}[P] \mathcal{M}\left(\|\varphi\|_{\infty}\right) d s .
$$

Letting $r=t-s$ in the integral gives

$$
\mid\left(G \varphi(t) \mid \leq \int_{0}^{\infty} M_{1} e^{-r} r^{-\beta}[P] \mathcal{M}(\|\varphi\|) d r\right.
$$

that is,

$$
|(G \varphi)(t)| \leq C_{1}(\beta) \mathcal{M}\left(\|\varphi\|_{\infty}\right),
$$

where $C_{1}(\beta)$ depends on $\beta, M_{1}, \epsilon$ and $[P]$. Next, for $t_{2}>t_{1}$, we have

$$
\begin{aligned}
\mid(G \varphi)( & \left.t_{2}\right)-(G \varphi)\left(t_{1}\right) \mid \\
\leq & \left|\left(\int_{-\infty}^{t_{2}}-\int_{-\infty}^{t_{1}}\right) T\left(t_{2}-s\right)(-A)^{\beta}(-A)^{-\beta} P(s) Q(\varphi(s)) d s\right| \\
& +\left|\int_{-\infty}^{t_{1}}\left(T\left(t_{2}-s\right)-T\left(t_{1}-s\right)\right)(-A)^{\beta}(-A)^{-\beta} P(s) Q(\varphi(s)) d s\right| \\
\leq & \int_{t_{1}}^{t_{2}}\left|T\left(t_{2}-s\right)(-A)^{\beta}(-A)^{-\beta} P(s) Q(\varphi(s))\right| d s \\
& +\int_{-\infty}^{t_{1}}\left|\left(T\left(t_{2}-t_{1}\right)-I\right) T\left(t_{1}-s\right)(-A)^{\beta}(-A)^{-\beta} P(s) Q(\varphi(s))\right| d s \\
= & J_{1}+J_{2} .
\end{aligned}
$$


By the same argument leading to (3.10) we get

$$
\begin{aligned}
J_{1} & \leq \int_{0}^{t_{2}-t_{1}} M_{1} e^{-\epsilon r} r^{-\beta}[P] \mathcal{M}\left(\|\varphi\|_{\infty}\right) d r \\
& \leq C_{2}(\beta) \mathcal{M}\left(\|\varphi\|_{\infty}\right)\left(t_{2}-t_{1}\right)^{1-\beta} .
\end{aligned}
$$

Also, we have

$$
\begin{aligned}
J_{2} \leq & \int_{-\infty}^{t_{1}} \mid\left(T\left(t_{2}-t_{1}\right)-I\right)(-A)^{-\gamma}\left(T\left(t_{1}-s\right)(-A)^{(\beta-\gamma)}(-A)^{-\beta} P(s) Q(\varphi(s)) \mid d s\right. \\
\leq & \int_{-\infty}^{t_{1}}\left|\left(T\left(t_{2}-t_{1}\right)-I\right)(-A)^{-\gamma}\right| \\
\leq & \left|\left(T\left(t_{2}-t_{1}\right)-I\right)(-A)^{-\gamma}\right| \\
& \quad \cdot \int_{-\infty}^{t_{1}}\left|T\left(t_{1}-s\right)(-A)^{(\beta-\gamma)}(-A)^{-\beta} P(s) Q(\varphi(s))\right| d s \\
\leq & \left|\left(T\left(t_{2}-t_{1}\right)-I\right)(-A)^{-\gamma}\right| C_{3}(\beta, \gamma) \mathcal{M}\left(\|\varphi\|_{\infty}\right)
\end{aligned}
$$

provided $0<\gamma<\beta$. Next recall that $(T(r)-I) g=\int_{0}^{r} T(s) A g d s$ for $g \in D(A)$, by the fundamental theorem of calculus. Thus, for $f \in \mathbb{Y}$,

$$
\begin{aligned}
\left|(T(r)-I)(-A)^{-\gamma} f\right| & =\left\|\int_{0}^{r} T(s)(-A)^{1-\gamma-\alpha}(-A)^{\alpha} f d s\right\| \\
& \leq\left\|(-A)^{\alpha} f\right\| \int_{0}^{r} M_{1} e^{-\epsilon s} s^{1-\gamma-\alpha} d s \\
& =C_{4}\left(\gamma, \epsilon, M_{1}\right) r^{2-\gamma-\alpha}|f|,
\end{aligned}
$$

since $1-\gamma-\alpha>-1$, because $0<\gamma<\beta<\alpha<1$.

In other words, $\left|(T(r)-I)(-A)^{-\gamma}\right| \leq C_{4} r^{2-\gamma-\alpha}$; consequently,

$$
J_{2} \leq C_{4}\left(t_{2}-t_{1}\right)^{2-\gamma-\alpha} C_{3} \mathcal{M}\left(\|\varphi\|_{\infty}\right) .
$$

For $\delta=\min (2-\gamma-\alpha, 1-\beta)>0$, it follows that

$$
\mid(G \varphi)\left(t_{2}\right)-\left(G \varphi\left(t_{1}\right)\left|\leq C_{5}\right| t_{2}-\left.t_{1}\right|^{\delta} \mathcal{M}\left(\|\varphi\|_{\infty}\right),\right.
$$

where $C_{5}$ depends on $\epsilon, M_{1},[P], \alpha, \beta, \gamma$ and $\mathbb{Y}$, that is, on parameters of the problem.

It follows that, for $\varphi \in B C(\mathbb{R}, \mathbb{X})$ with $\|\varphi(t)\| \leq R$ for all $t \in \mathbb{R}$, then $G \varphi \in$ $B C^{\delta}(\mathbb{R}, \mathbb{Y})$ with $\|G \varphi(t)\| \leq R_{1}$ for all $t \in \mathbb{R}$ and some $R_{1}$ that depends on $R$. This completes the proof.

Proposition 3.2. The function $G$ maps bounded sets of $A A(\mathbb{X})$ into bounded sets of $B C^{\delta}(\mathbb{R}, \mathbb{Y}) \cap A A(\mathbb{X})$ for $0<\delta<\alpha$.

Proof. We just need to check that

$$
G(A A(\mathbb{X})) \subset A A(\mathbb{X}) .
$$

To this end, let $\varphi \in A A(\mathbb{X})$. Then given a sequence $\left(s_{n}^{\prime}\right) \subset \mathbb{R}$, there exists a subsequence $\left(s_{n}\right) \subset\left(s_{n}^{\prime}\right)$ such that

$$
\psi(t)=\lim _{n \rightarrow \infty} \varphi\left(t+s_{n}\right)
$$

is well defined for each $t \in \mathbb{R}$ and

$$
\lim _{n \rightarrow \infty} \psi\left(t-s_{n}\right)=\varphi(t)
$$


for each $t \in \mathbb{R}$. Since $\psi \in B C(\mathbb{R}, \mathbb{X})$, then

$$
(G \varphi)\left(t+s_{n}\right)=\int_{-\infty}^{t+s_{n}} T\left(t+s_{n}-s\right) P(s) Q(\varphi(s)) d s .
$$

Let $\sigma=s-s_{n}$. Then

$$
\begin{aligned}
(G \varphi)\left(t+s_{n}\right) & =\int_{-\infty}^{t} T(t-\sigma) P\left(\sigma+s_{n}\right) Q\left(\varphi\left(\sigma+s_{n}\right)\right) d \sigma \\
& =\int_{-\infty}^{t} T(t-\sigma) P_{n}(\sigma) Q_{n}(\sigma) d \sigma
\end{aligned}
$$

where $P_{n}(\sigma)=P\left(\sigma+s_{n}\right), Q_{n}(\sigma)=Q\left(\varphi\left(\sigma+s_{n}\right)\right), n=1,2, \cdots, \sigma \in \mathbb{R}$.

Since $P \in A A(\mathbb{Z})$, there exists a subsequence of $\left(s_{n}\right)$, which we still denote by $\left(s_{n}\right)$, such that

$$
\hat{P}(\sigma)=\lim _{n \rightarrow \infty} P_{n}(\sigma)
$$

exists for each $\sigma \in \mathbb{R}$ and

$$
\lim _{n \rightarrow \infty} \hat{P}\left(\sigma-s_{n}\right)=P(\sigma)
$$

for each $\sigma \in \mathbb{R}$. Clearly we also have, by passing to a subsequence if necessary,

$$
\lim _{n \rightarrow \infty} \varphi\left(t+s_{n}\right)=\psi(t)
$$

and

$$
\lim _{n \rightarrow \infty} \psi\left(t-s_{n}\right)=\varphi(t)
$$

for each $t \in \mathbb{R}$. By the Bochner integral version of Lebesgue's dominated convergence theorem, we get

$$
\begin{aligned}
& (G \varphi)\left(t+s_{n}\right)=\int_{-\infty}^{t} T(t-\sigma) P_{n}(\sigma) Q_{n}(\sigma) d \sigma \\
& \longrightarrow \int_{-\infty}^{t} T(t-\sigma) \hat{P}(\sigma) Q(\varphi(\sigma)) d \sigma=\chi(t)
\end{aligned}
$$

for each $t \in \mathbb{R}$, and

$$
\begin{aligned}
\chi\left(t-s_{n}\right) & =\int_{-\infty}^{t-s_{n}} T\left(t-s_{n}-\sigma\right) \hat{P}(\sigma) Q(\psi(\sigma)) d \sigma \\
& =\int_{-\infty}^{t} T(t-r) \hat{P}\left(r-s_{n}\right) Q\left(\psi\left(r-s_{n}\right)\right) d r
\end{aligned}
$$

by letting $r=\sigma+s_{n}$. Thus we obtain

$$
\chi\left(t-s_{n}\right) \longrightarrow \int_{-\infty}^{t} T(t-r) P(r) Q(\varphi(r)) d r=(G \varphi)(t),
$$

again by Lebesgue's dominated convergence theorem. This shows that $G(A A(\mathbb{X}))$ $\subset A A(\mathbb{X})$, and the proof is now complete.

Proposition 3.3. $B C^{\delta}(\mathbb{R}, \mathbb{Y})$ is compactly contained in $B C(\mathbb{R}, \mathbb{X})$; in other words, the canonical injection id $: B C^{\delta}(\mathbb{R}, \mathbb{Y}) \rightarrow B C(\mathbb{R}, \mathbb{X})$ is compact, which implies that

$$
i d: B C^{\delta}(\mathbb{R}, \mathbb{Y}) \cap A A(\mathbb{X}) \rightarrow A A(\mathbb{X})
$$

is compact too. 
Proof. We show that $i d$ maps bounded sets of $B C^{\delta}(\mathbb{R}, \mathbb{Y})$ into relatively compact sets of $B C(\mathbb{R}, \mathbb{X})$. To this end, let $\left(\varphi_{\nu}\right)$ be a bounded sequence in $B C^{\delta}(\mathbb{R}, \mathbb{Y})$. Let $\mathbb{Q}=\left\{r_{n}\right\}$ be the set of all rational numbers. Then $\left(\varphi_{\nu}\left(r_{n}\right)\right)$ is a bounded sequence in $\mathbb{Y}$, for each $n$. By the well-known Cantor diagonalization process, there exists a subsequence $\left(\varphi_{\nu_{k}}\right)$ such that

$$
\varphi_{\nu_{k}}\left(r_{n}\right) \rightarrow \varphi\left(r_{n}\right)
$$

as $k \rightarrow \infty$ in $\mathbb{X}$, for each $n$, and some $\varphi: \mathbb{Q} \rightarrow \mathbb{X}$. But the sequence $\left(\varphi_{n}\right)$ is an equicontinuous family in $B U C(\mathbb{R}, \mathbb{Y}) \subset B U C(\mathbb{R}, \mathbb{X})$, because of the uniform Hölder condition. Thus, as in the proof of the Arzela-Ascoli theorem, there is a further subsequence (which we still denote by $\left(\varphi_{\nu_{k}}\right)$ ) satisfying

$$
\varphi_{\nu_{k}}(t) \rightarrow \varphi(t) \text {, as } k \rightarrow \infty
$$

in $\mathbb{X}$, for all $t \in \mathbb{R}$. In addition the convergence is uniform in $t \in \mathbb{R}$. Note that $B U C(\mathbb{R}, \mathbb{X})$ can be identified with $C(K, \mathbb{X})$ for a suitable Hausdorff compactification $K$ of $\mathbb{R}$ (see for instance [3]). Thus the convergence $\varphi_{\nu_{k}} \rightarrow \varphi$ holds in $B U C(\mathbb{R}, \mathbb{X}) \subset$ $B C(\mathbb{R}, \mathbb{X})$. This completes the proof.

Proposition 3.4. The function $G$ has a fixed point in $A A(\mathbb{X})$.

Proof. Let us recall that the estimates (3.10)-(3.11), $|G \varphi|_{\infty} \leq C_{1}(\beta) \mathcal{M}\left(\|\varphi\|_{\infty}\right)$ and $\mid(G \varphi)\left(t_{2}\right)-\left(G \varphi\left(t_{1}\right)\left|\leq C_{5}\right| t_{2}-t_{1} \mid \delta \mathcal{M}\left(\|\varphi\|_{\infty}\right)\right.$, hold for all $\varphi \in B C(\mathbb{R}, \mathbb{Y})$ and all $t_{1}, t_{2} \in \mathbb{R}$ with $t_{2}$ not equal to $t_{1}$. It follows that there exists a constant $C_{6}=C_{6}\left(\epsilon, M, M_{1}, \alpha, \beta, \gamma\right)$ such that

$$
\begin{aligned}
\varphi \in B C(\mathbb{R}, \mathbb{X}) & \text { and } \quad\|\varphi\|_{\infty}<R \text { imply } \\
G \varphi \in B C^{\delta}(\mathbb{R}, \mathbb{Y}) & \text { and } \quad|G \varphi|<R_{1},
\end{aligned}
$$

where $R_{1}=C_{6} \mathcal{M}(R)$.

Since $\mathcal{M}(R) / R \rightarrow 0$ as $R \rightarrow \infty$, and since $\|y\| \leq C_{7}|y|$ holds for some constant $C_{7}$ and all $y \in \mathbb{Y}$, it follows that there exists $\rho>0$ such that for all $R \geq \rho$, we have

$$
G\left(B_{A A(\mathbb{X})}(0, R)\right) \subset B_{B C^{\delta}(\mathbb{R}, \mathbb{Y})}(0, R) \cap B_{A A(\mathbb{X})}(0, R) .
$$

Since $G$ leaves $A A(\mathbb{X}) \subset B C(\mathbb{R}, \mathbb{X})$ invariant, the estimate (3.13) along with the continuity properties of $G$ imply that $G$ is a continuous, compact mapping $S \rightarrow S$, where $S$ is the ball of radius $R$ in $A A(\mathbb{X})$ and $R \geq \rho$. By the Schauder fixed point theorem, $G$ has a fixed point in $S, \varphi_{0}$. Obviously, $\varphi_{0}$ is a mild solution of (1.1).

Finally, the above results can be summarized as follows.

Theorem 3.5. Let $A$ generate an exponentially stable $C_{0}$-semigroup $\mathcal{T}$ in $\mathcal{B}(\mathbb{X})$. Assume assumptions (1.1) and (3.2)-(3.5). Then (1.1) has a mild solution in $A A(\mathbb{X})$.

Now we end this paper with the following

Example of nonuniqueness. Let $\mathbb{X}=\mathbb{R}, A=-1$ and

$$
u(t)= \begin{cases}t^{3 / 2} e^{1-t}, & \text { for } t \in\left[0, \frac{3}{2}\right], \\ 0, & \text { for } t \in\left[-\frac{3}{2}, 0\right] .\end{cases}
$$

Then for $t \in\left[0, \frac{3}{2}\right]$ we have

$$
u^{\prime}(t)=-u(t)+\frac{3}{2} t^{1 / 2} e^{(1-t)}=-u(t)+\frac{3}{2} u(t)^{1 / 3} e^{\frac{2}{3}(1-t)}=-u(t)+f(t, u(t))
$$


where

$$
f(t, \varphi)= \begin{cases}\frac{3}{2} \varphi^{1 / 3} e^{\frac{2}{3}(1-t)}, & \text { for } t \in\left[0, \frac{3}{2}\right] \times \mathbb{R}, \\ \frac{3}{2} \varphi^{1 / 3} e^{2 / 3}, & \text { for } t \in\left[-\frac{3}{2}, 0\right] \times \mathbb{R} .\end{cases}
$$

Note that $u^{\prime}\left(\frac{3}{2}\right)=0$ and $u\left(\frac{3}{2}\right)=\left(\frac{3}{2}\right)^{\frac{3}{2}} e^{-\frac{3}{2}}$.

Now let $f(t, \varphi)=f\left(\frac{3}{2}, \varphi\right)$ on $\left[\frac{3}{2}, 3\right] \times \mathbb{R}$ and $f(t, \varphi)=f\left(\frac{9}{2}-t, \varphi\right)$ on $\left[3, \frac{9}{2}\right] \times \mathbb{R}$; let $u(t)=u\left(\frac{3}{2}\right)$ on $\left[\frac{3}{2}, 3\right]$, and $u(t)=u\left(\frac{9}{2}-t\right)$ on $\left[3, \frac{9}{2}\right]$. Then $u^{\prime}=-u+f(t, u)$ on $\left[-\frac{3}{2}, \frac{9}{2}\right]$, together with $u(0)=0$.

Extend $u$ to be a periodic function of period 6 (hence an almost automorphic function). Then $u$ and $v \equiv 0$ both satisfy

$$
\frac{d x}{d t}=-x+f(t, x), \quad x(0)=0 .
$$

\section{REFERENCES}

[1] S. Bochner, Continuous mappings of almost automorphic and almost periodic functions, Proc. Nat. Acad. Sci. USA 52(1964), 407-410. MR0168997 (29:6252)

[2] T. Diagana, G. M. N'Guérékata and N. V. Minh, Almost automorphic solutions of evolution equations, Proc. Amer. Math. Soc. 132 (2004), 3289-3298. MR 2073304

[3] N. Dunford and J. T. Schwartz, Linear Operators. Vol. I, Interscience, New York, 1964. MR 0117523(22:8302)

[4] J. A. Goldstein, Semigroups of Linear Operators and Applications, Oxford University Press, Oxford, 1985. MR0790497 (87c:47056)

[5] G. M. N'Guérékata, Existence and uniqueness of almost automorphic mild solutions to some semilinear abstract differential equations, Semigroup Forum 69 (2004), no. 1, 80-86. MR 2063980

[6] G. M. N'Guérékata, Almost Automorphic and Almost Periodic Functions in Abstract Spaces, Kluwer Academic/ Plenum Publishers, New York, 2001. MR1880351 (2003d:43001)

Department of Mathematical Sciences, University of Memphis, Memphis, Tennessee 38152-3240

E-mail address: jgoldste@memphis.edu

Department of Mathematics, Morgan State University, Baltimore, Maryland 21251

E-mail address: gnguerek@jewel.morgan.edu 